\author{
S.D. Maussumbekova iD A. Duysenali* \\ Al-Farabi Kazakh National University \\ *e-mail: duisenali_akzhol1@live.kaznu.kz
}

\title{
Numerical simulation of boiling liquid outflows process
}

\begin{abstract}
Computational algorithm was developed based on the well-known ANSYS Fluent software package for studying unsteady wave outflow of a saturated liquid from high-pressure pipelines during emergency depressurization. The unsteady processes of the outflow of liquid, boiling as a result of depressurization of high-pressure vessels, have been investigated. The system of equations for the conservation of mass, momentum, and energy in a two-dimensional coordinate system is used in order to create mathematical model this process. The features of the formation of jets of boiling liquid at various equilibrium initial states of water in a high-pressure vessel under conditions close to the experiments carried out by the authors [10] have been studied. The spatial distributions of pressure, temperature and velocity of the forming jet are obtained. Numerical results have shown that, over time, the character of the velocity distribution acquires a conical shape. With an increase in the initial temperature and pressure, this distribution is preserved, and the opening angle increases. The results are in qualitative agreement with the experimental data.
\end{abstract}

Key words: outflows of boiling liquid, mathematical and numerical modeling, homogeneous mixture, depressurization of a pressure vessel.

\section{Intraduction}

The relevance of studies of the wave process of the efflux of a boiling coolant is due to the increasing requirements for ensuring the safety of modern power plants in emergency operating conditions. Establishing the laws of changing the parameters of the coolant in time in high-pressure circuits during sudden depressurization is necessary for calculating additional loads and extreme temperatures in the structural elements of the circuits, for designing devices for localizing the consequences of an accident, etc. In engineering practice, the calculation of the emergency outflow of a high-temperature coolant is usually carried out within the framework of quasi-stationary methods [1-4].

An experimental study of the dynamics of a superheated liquid from high-pressure chambers was carried out in $[5,6]$. Numerical modeling of the process of depressurization of a pipeline with a hot coolant on the basis of a homogeneous model was carried out in [7]. On the basis of experiments from [5 non-stationary processes of efflux of boiling water in a one-dimensional formulation were numerically investigated in [8]. The authors generalized the twophase model of the boiling steam-water mixture for the two-dimensional case with axial symmetry in $[9,10]$. The features of the formation of jets of a boiling liquid were studied at various values of the initial saturation parameters close to the thermodynamic critical point.

Numerical modeling of the process of formation of a jet of a boiling coolant taking into account the nonequilibrium of the steam-water mixture near the rupture boundary is presented in [8]. The authors had used the model of an ideal compressible fluid in the quasi-stationary approximation in order to solve the problem. The experiments on explosive boiling up of water jets as a result of their outflow through a thin cylindrical nozzle from high-pressure vessels are analyzed in [10].

The study of the motion of heterogeneous mixtures taking into account the initial structure of the mixture and the physical properties of the phases is associated with the use of new parameters and the solution of equations that are more complicated than those with which one has to deal with in the mechanics of single-phase (homogeneous) media. In addition, a detailed description of intraphase and interphase interactions in heterogeneous media is extremely difficult. Rational schematization is especially necessary here to obtain observable results and their understanding.

The works [5] - [11] are devoted to the issues of the expiration of two-phase flows. High values of temperature and pressure in containers with water

$$
\text { Int. j. math. phys. (Online) }
$$


during depressurization lead to its rapid expansion and boiling. The initial, unsteady stage of the efflux of a boiling liquid is of particular interest both in theoretical and experimental terms, since it is characterized by significant metastable wave processes.

The problem of emergency depressurization of systems with a liquid coolant at high pressure is far from well understood. This also applies to the mechanical side of the phenomenon of wave outflow of a boiling liquid, accompanied by intense phase transformations and restructuring of the structure of the vapor-liquid flow. It is advisable to carry out theoretical research in the direction of analyzing the physics of the process, constructing its mathematical models and developing methods for numerical integration that describe the process of equations.

\section{Formulation of the problem}

The main assumptions were used in the mathematical formulation of the problem like:

- the movement of the medium is twodimensional, the influence of the design features of the pipelines on the outflow process can be neglected;

- the temperature inside the pipe is identically equal to the saturation temperature at a given pressure.

The equations of state of this medium are constructed according to the known equations of state of the phases using the usual assumption about the additivity of thermodynamic functions:

$$
\begin{aligned}
i(x, p) & =(1-x) i_{1}(p)+x i_{2}(p)= \\
& =i_{1}(p)+x l(p) \\
V(p) & =(1-x) V_{1}(p)+x V_{2}(p)
\end{aligned}
$$

there $i$ и $V$ - specific enthalpy and volume of the mixture; $l$ - specific heat of vaporization; indices 1 and 2 mark the parameters of liquid and vapor, respectively. The equations of conservation of mass and momentum and the equation of heat inflow for two-dimensional unsteady motion of an equilibrium vapor-liquid mixture in a channel of constant crosssection have the following form:

$$
\frac{d \rho}{d t}+\frac{\rho \partial v_{x}}{\partial x}+\frac{\rho \partial v_{y}}{\partial y}=0
$$

$$
\begin{gathered}
\rho \frac{d v_{x}}{d t}=-\frac{\partial p}{\partial x}, \quad \rho \frac{d v_{y}}{d t}=-\frac{\partial p}{\partial y} \\
\rho \frac{d e}{d t}+p \operatorname{div} v=0
\end{gathered}
$$

Let there be a cylindrical vessel (a piece of pipeline) of constant cross-section with a length $L$, filled with a saturated liquid with pressure $p_{0}$ and temperature $T_{s}\left(p_{0}\right)$. At the moment of time $\mathrm{t}=0$, a sudden depressurization of the right end occurs (rupture of the diaphragm). The liquid in the outlet section boils, the forming vapor-liquid mixture starts to move and flows out with a pressure $p_{c}$, , much less than the pressure $p_{0}$. A rarefaction wave will go through the liquid inside the pipe. The problem of calculating a non-stationary process accompanying a sudden depressurization of a pipeline is to determine the laws of change in pressure, velocity, steam content and temperatures in time in various sections of the pipe. Mathematically, it is a mixed problem for the system of partial differential equations (2) - (4) with the following initial and boundary conditions:

$$
\begin{gathered}
\vartheta(z, 0)=0, \quad p(z, 0)=p_{0}, \\
T(z, 0)=T_{s}\left(p_{0}\right) \\
\vartheta(0, t)=0, \quad p(L, t)=p_{c}
\end{gathered}
$$

\section{Numerical solution technique}

ANSYS software was used for solving of problem. At first stage, geometry of object was created with the help of built-in DesignModeler editor. Mesh editor allows sampling computational domain. Transition to Setup editor allows setting initial and boundary conditions of task, and choose solution method. (Figure 1).

The geometry of the computational domain is a rectangular section of the pipe, which corresponds to the following dimensions: the part of the section where the boiling liquid is located: length $-7 \mathrm{~cm}$, width $-1 \mathrm{~cm}$, and the medium - air: length. $-14 \mathrm{~cm}$, width $-2 \mathrm{~cm}$, the full computational domain is rectangular with length $-21 \mathrm{~cm}$ and height $3 \mathrm{~cm}$. The algorithm for creating the geometry and mesh of the problem, setting the boundary conditions in the Ansys software are shown in Figures 2-3. 
schematic

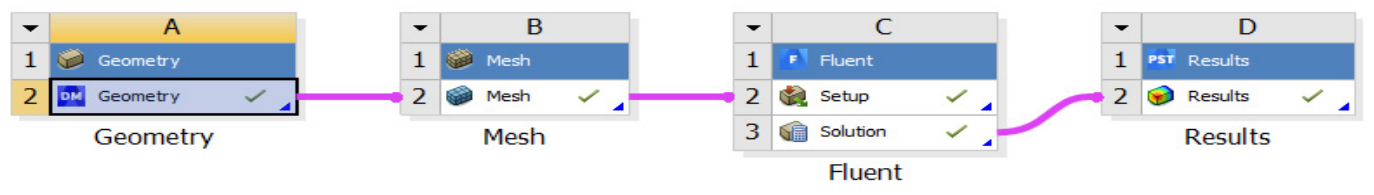

Figure 1 - Ansys project

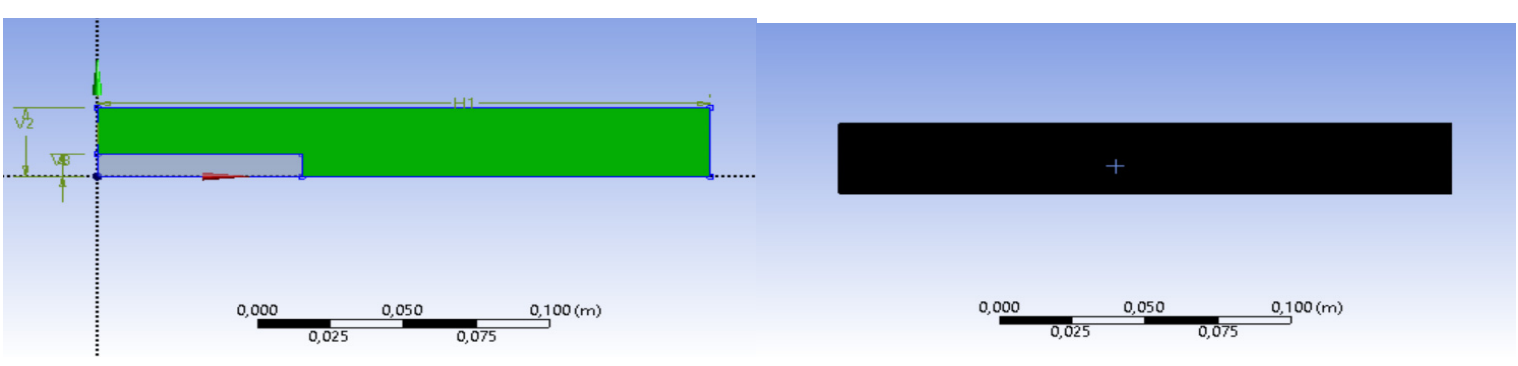

Figure 2 - Geometry of the computational domain

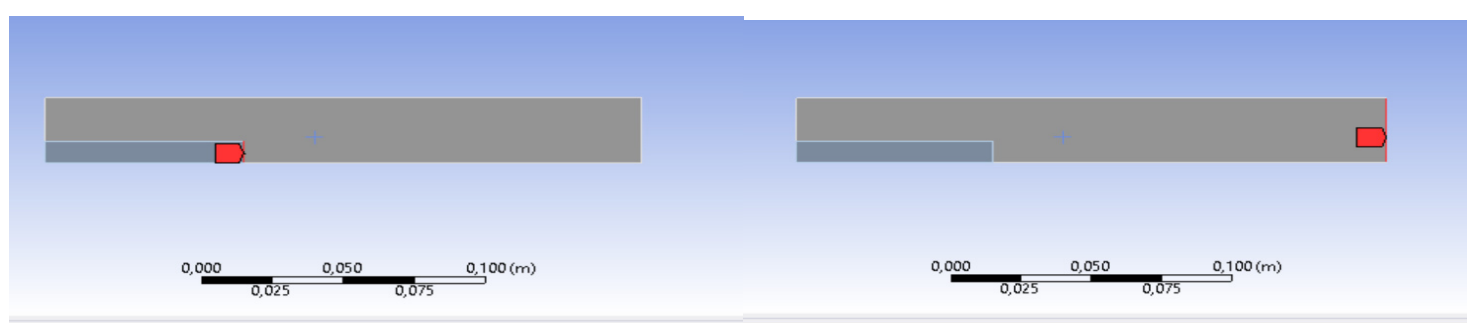

a

b

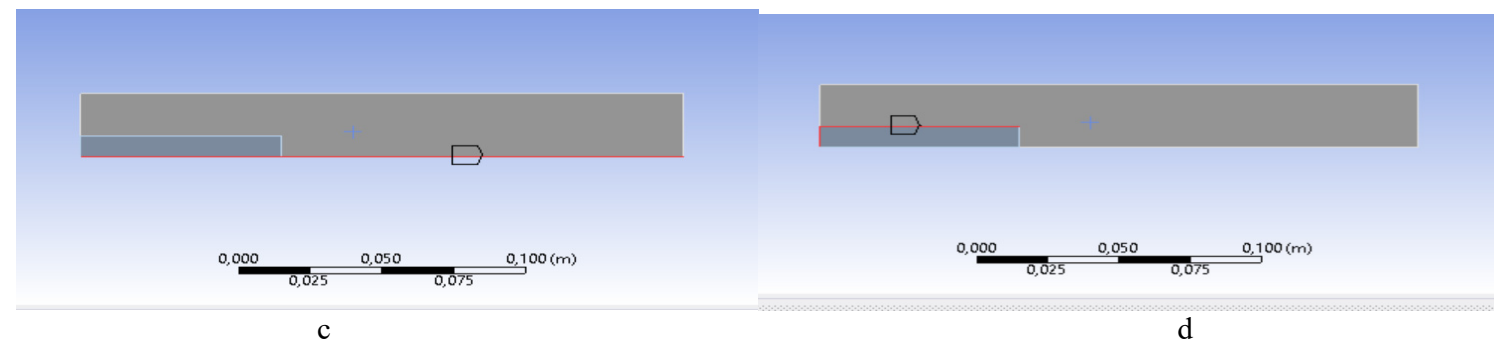

Figure 3 - Boundaries of the computational domain: Inlet (a); Outlet (b); Axis (c); Pipe Wall (d)

The boundary conditions are set as follows: the air velocity in the free volume is $0.001 \mathrm{~m} / \mathrm{s}$. The relative pressure at the outlet from the pipe is $0 \mathrm{~Pa}$. To obtain a solution, we choose a paired calculation scheme that combines pressure and speed.

\section{Results and analysis of numerical calculations}

We investigated the dynamics of the vapor-liquid mixture during depressurization of the pipe under various initial conditions inside the pipe. The following values were set as the initial values for the pressure and temperature inside the pipe: A: $p=$ 246 кПа, $T=400 K$; В: $p=475$ кПа, $T=423 K$; C: $p=1553$ кПа, $T=473 \mathrm{~K}$ and $T=300 \mathrm{~K}$ in outside zone.

The pressure and velocity profiles are shown in figure 4 for various pipe sections after depressurization. Numerical results lead that the mixture instantly sets in motion: the speed increases, the pressure 
drops. The closer the discontinuity point is $(7 \mathrm{~cm})$, the more intense the pressure drop, which is consistent with the results of [8].

The pressure contours are shown in Figure 5 at different times with initial values $p=246$ кПа, $T=400 K$. As a result of depressuri- -zation, a fast unloading wave propagates inside the pipe, and a spatial outflow of the boiling flow begins, in which a radial expansion of the jet is observed due to vaporization.

The velocity profiles acquire a characteristic conical shape with changing time.
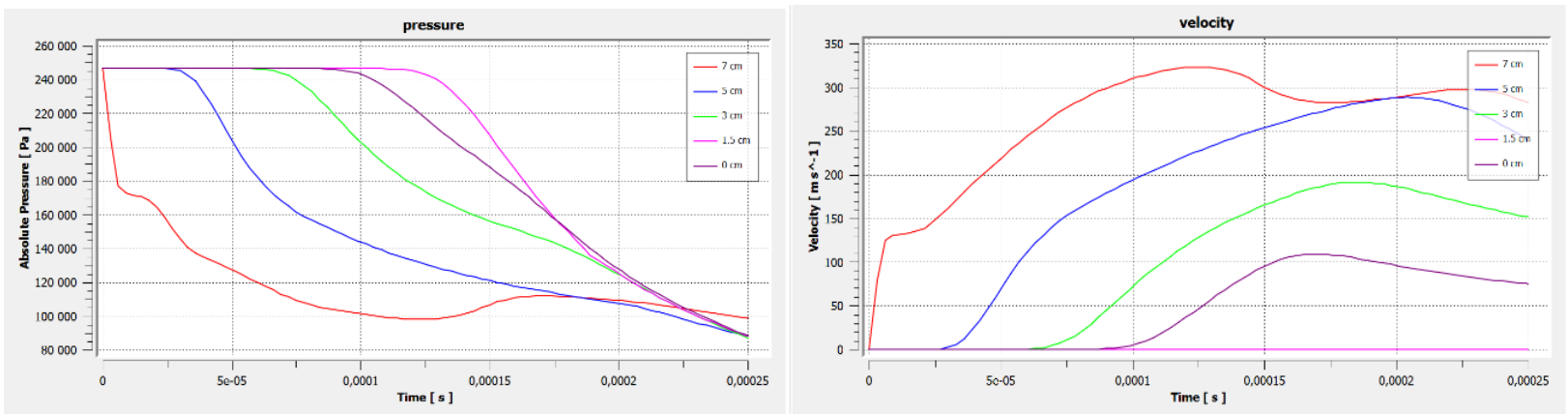

B
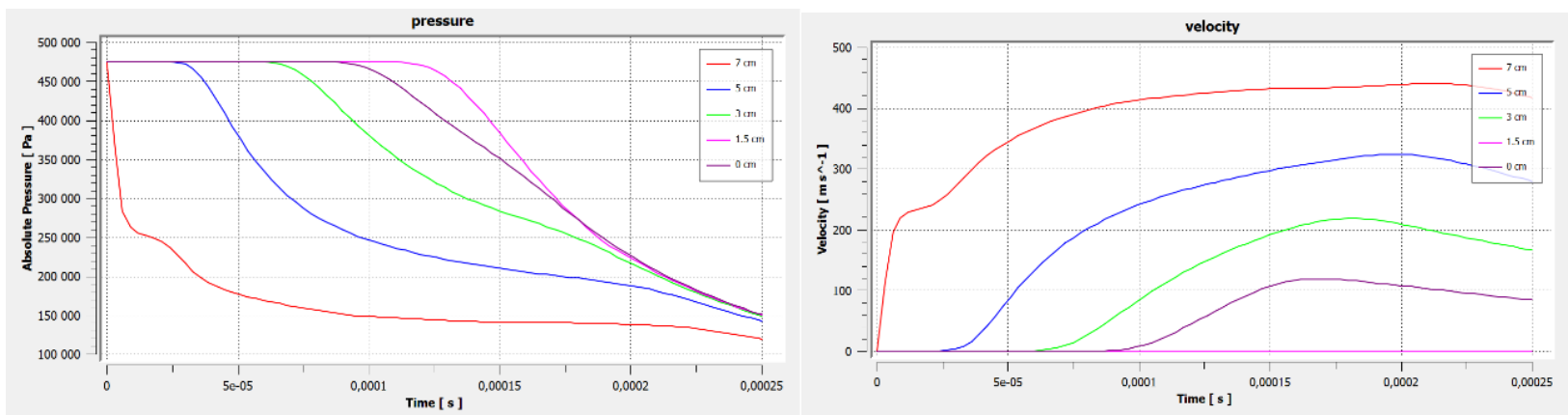

C
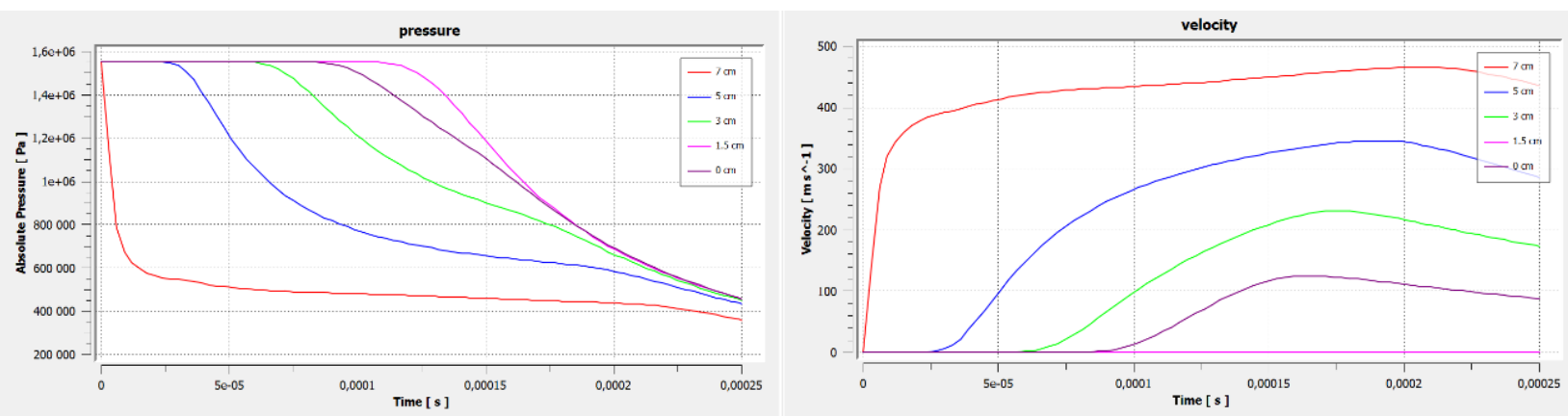

Figure 4 - Distribution of pressure (left) and velocity (right) under different initial conditions in the pipe:
A: $p=246$ кПа, $T=400 K$
В: $p=475$ кПа, $T=423 K$;
С: $p=1553$ кПа, $T=473 K$. 
The stream of boiling water also has a conical shape, and the opening angle increases with increasing in the initial temperature and pressure in the saturation state to $423 \mathrm{~K}$ and $475 \mathrm{kPa}$, respectively (Figure 6) in comparison with the previous version.

a

\begin{tabular}{|c|c|}
\hline $\begin{array}{l}\text { Abşolute Pressure } \\
2.467 \mathrm{e}+05 \\
2.290 \mathrm{e}+05 \\
2.114 \mathrm{e}+05 \\
1.938 \mathrm{e}+05 \\
1.762 \mathrm{e}+05 \\
1.586 \mathrm{e}+05 \\
1.409 \mathrm{e}+05 \\
1.233 \mathrm{e}+05 \\
1.057 \mathrm{e}+05 \\
8.809 \mathrm{e}+04 \\
7.047 \mathrm{e}+04 \\
{[\mathrm{~Pa}]}\end{array}$ & $\begin{array}{l}\text { a } \\
\text { Velocity } \\
2.842 \mathrm{e}+02 \\
2.557 \mathrm{e}+02 \\
2.273 \mathrm{e}+02 \\
1.989 \mathrm{e}+02 \\
1.705 \mathrm{e}+02 \\
1.421 \mathrm{e}+02 \\
1.137 \mathrm{e}+02 \\
8.525 \mathrm{e}+01 \\
5.683 \mathrm{e}+01 \\
2.842 \mathrm{e}+01 \\
0.000 \mathrm{e}+00 \\
\left.\left[\mathrm{~m} \mathrm{~s}^{-}-1\right]\right]\end{array}$ \\
\hline
\end{tabular}

b

\begin{tabular}{|c|c|}
\hline $\begin{array}{l}2.467 e+05 \\
2.290 e+05\end{array}$ & $\begin{array}{l}2.842 \mathrm{e}+02 \\
2.557 \mathrm{e}+02\end{array}$ \\
\hline $2.114 e+05$ & $2.273 e+02$ \\
\hline $1.938 e+05$ & $1.989 e+02$ \\
\hline $1.762 \mathrm{e}+05$ & $1.705 e+02$ \\
\hline $1.586 \mathrm{e}+05$ & $1.421 \mathrm{e}+02$ \\
\hline $1.409 e+05$ & $1.137 \mathrm{e}+02$ \\
\hline $1.233 e+05$ & $8.525 \mathrm{e}+01$ \\
\hline $\begin{array}{l}1.057 e+05 \\
8.809 e+04\end{array}$ & $\begin{array}{l}5.683 e+01 \\
2.842 e+01\end{array}$ \\
\hline$[\mathrm{Pa}] .047 \mathrm{e}+04$ & 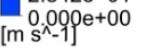 \\
\hline
\end{tabular}

c

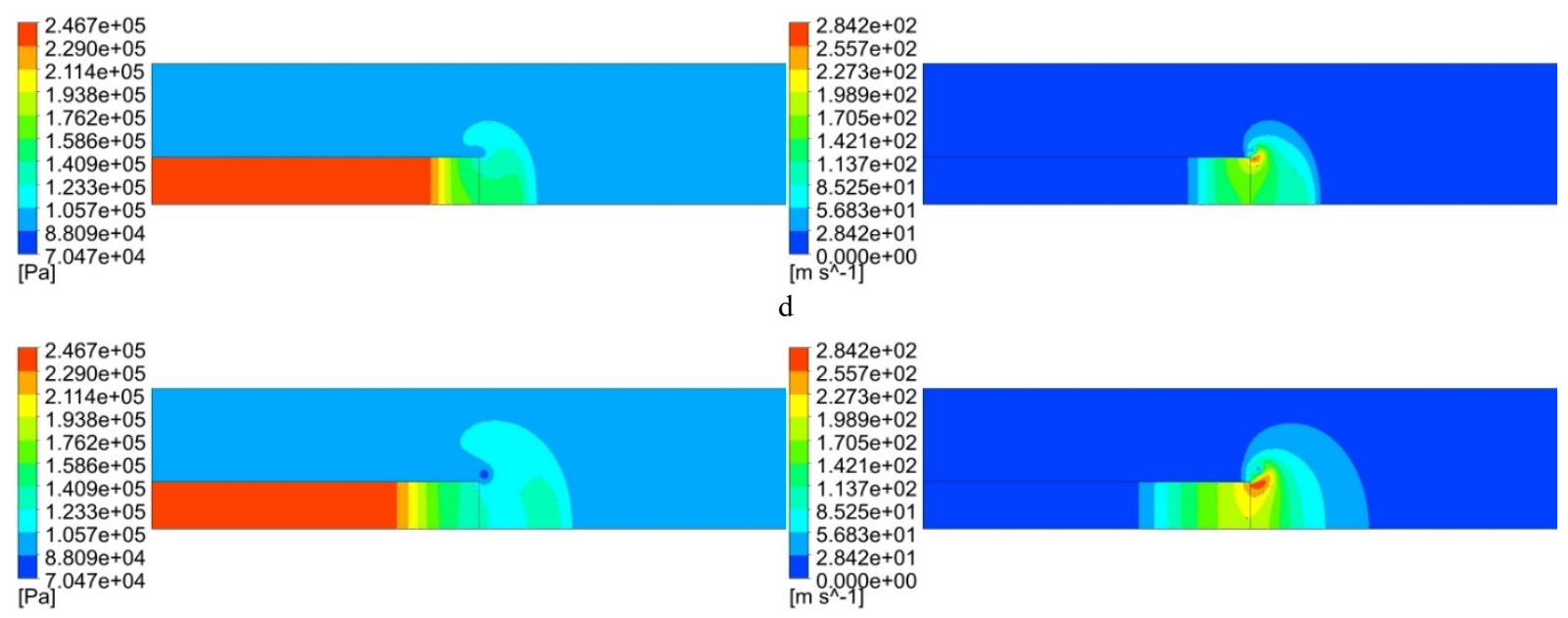

Figure 5 - Pressure (left) and velocity (right) circuits at different points in time: $\mathrm{t}=0 \mathrm{c}(\mathrm{a}) ; \mathrm{t}=1.5 \mathrm{e}-5 \mathrm{c}(\mathrm{b}) ; \mathrm{t}=2.4 \mathrm{e}-5 \mathrm{c}(\mathrm{c}) ; \mathrm{t}=4.2 \mathrm{t}-5 \mathrm{c}(\mathrm{d})$ for $p=246.7$ кПа, $T=400 K$; 


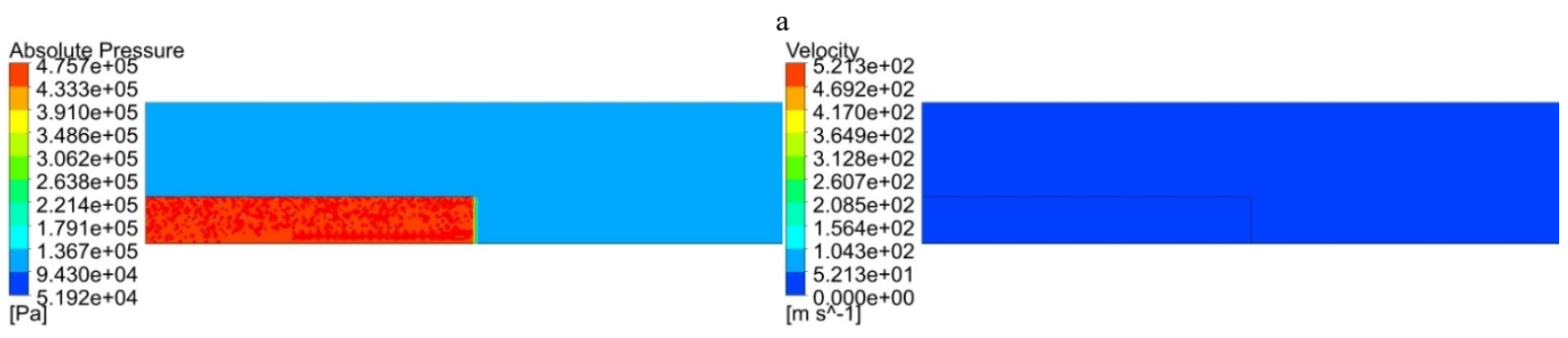

b

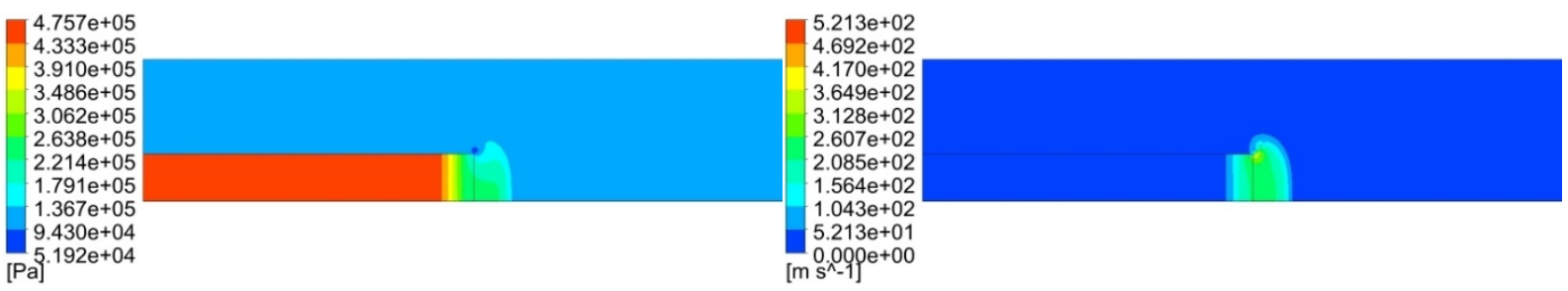

c

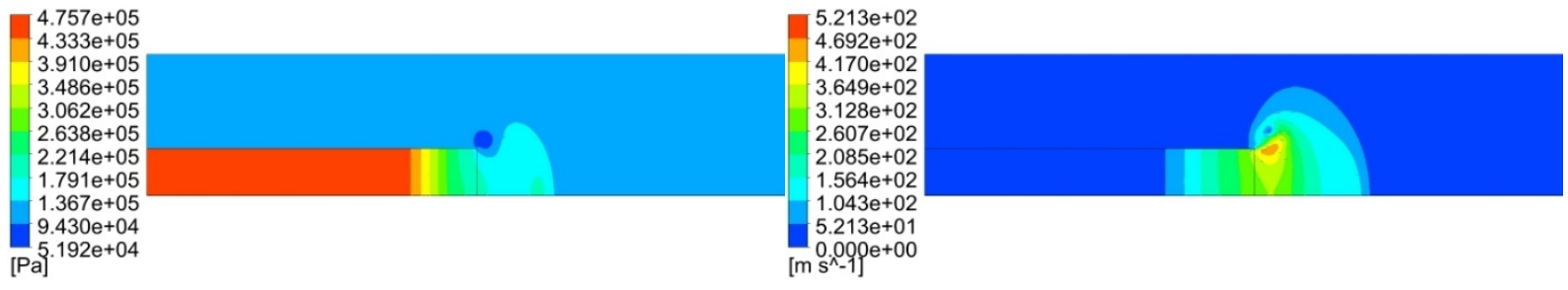

d

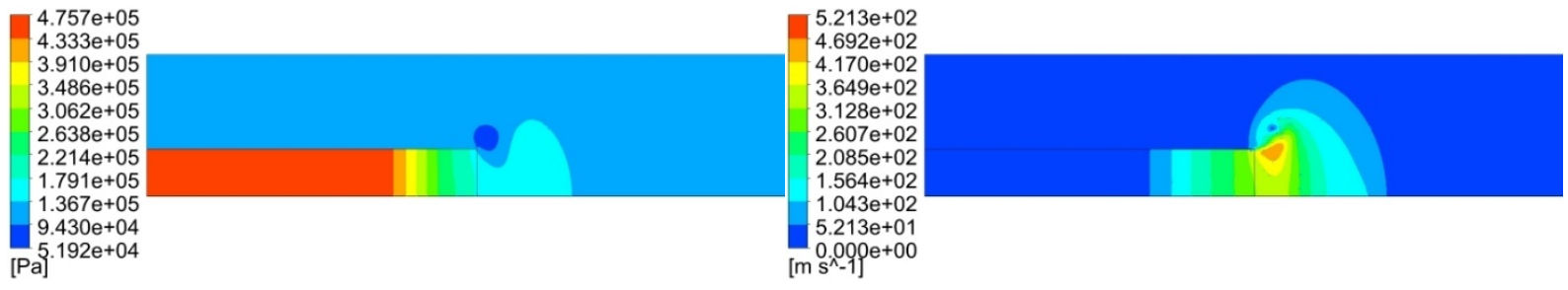

Figure 6 - Pressure (left) and velocity (right) circuits at different points in time:

$\mathrm{t}=0 \mathrm{c}(\mathrm{a}) ; \mathrm{t}=1.5 \mathrm{e}-5 \mathrm{c}(\mathrm{b}) ; \mathrm{t}=2.4 \mathrm{e}-5 \mathrm{c}(\mathrm{c}) ; \mathrm{t}=4.2 \mathrm{t}-5 \mathrm{c}(\mathrm{d})$

for $p=475$ кПа, $T=423 \mathrm{~K}$

Calculations have shown that a further increase in temperature to $473 \mathrm{~K}$ leads to a significant increase in speed, but the jet opening angle does not exceed 70 degrees (Figure 7). This result is consistent with the experimental data from [10], in which, up to the indicated temperatures, the jet also had a conical shape with an increase in the opening angle with an increase in the initial temperature. 


Absolute Pressure
$1.554 \mathrm{e}+06$
$1.402 \mathrm{e}+06$
$1.251 \mathrm{e}+06$
$1.100 \mathrm{e}+06$
$9.482 \mathrm{e}+05$
$7.968 \mathrm{e}+05$
$6.455 \mathrm{e}+05$
$4.941 \mathrm{e}+05$
$3.428 \mathrm{e}+05$
$1.914 \mathrm{e}+05$
$4.008 \mathrm{e}+04$

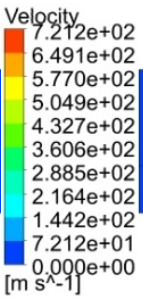

b

$1.554 \mathrm{e}+06$

$1.402 \mathrm{e}+06$

$1.251 e+06$
$1.100 e+06$

$1.100 \mathrm{e}+06$

$7.968 \mathrm{e}+05$

$6.455 \mathrm{e}+05$

$4.941 \mathrm{e}+05$

$3.428 \mathrm{e}+05$

$1.914 \mathrm{e}+05$

$4.008 \mathrm{e}+04$

$7.212 \mathrm{e}+02$

$6.491 \mathrm{e}+02$

$5.770 e+02$
$5.049 e+02$

$5.049 e+02$
$4.327 e+02$

$4.327 \mathrm{e}+02$

$3.606 \mathrm{e}+02$
$2.885 \mathrm{e}+02$

$2.885 \mathrm{e}+02$
$2.164 \mathrm{e}+02$

$2.164 \mathrm{e}+02$
$1.442 \mathrm{e}+02$

$1.442 \mathrm{e}+02$
$7.212 \mathrm{e}+01$

$0.000 \mathrm{e}+00$

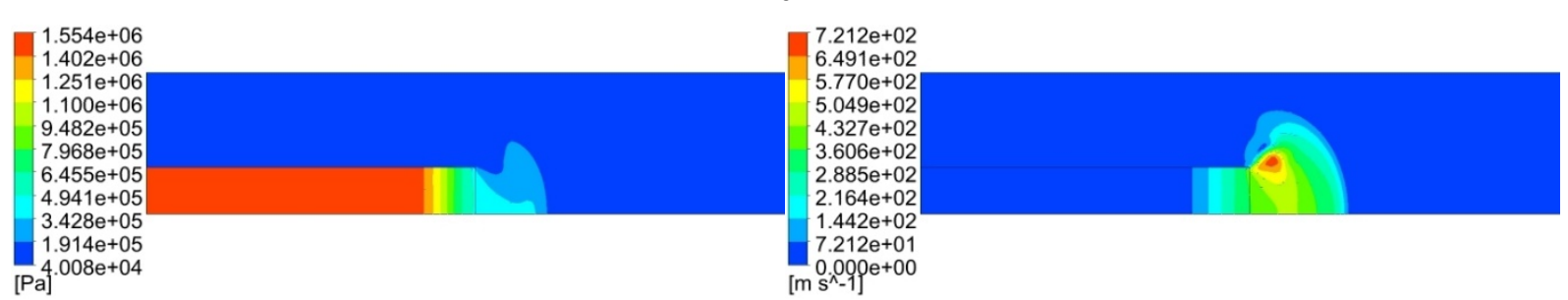

d

\begin{tabular}{|l|l}
$1.554 \mathrm{e}+06$ & $7.338 \mathrm{e}+02$ \\
$1.402 \mathrm{e}+06$ & $6.604 \mathrm{e}+02$ \\
$1.251 \mathrm{e}+06$ & $5.870 \mathrm{e}+02$ \\
$1.100 \mathrm{e}+06$ & $5.137 \mathrm{e}+02$ \\
$9.482 \mathrm{e}+05$ & $4.403 \mathrm{e}+02$ \\
$7.968 \mathrm{e}+05$ & $3.669 \mathrm{e}+02$ \\
$6.455 \mathrm{e}+05$ & $2.935 \mathrm{e}+02$ \\
$4.941 \mathrm{e}+05$ & $2.201 \mathrm{e}+02$ \\
$3.428 \mathrm{e}+05$ & $1.468 \mathrm{e}+02$ \\
$1.914 \mathrm{e}+05$ & $7.338 \mathrm{e}+01$ \\
\hline $4.008 \mathrm{e}+04$ & $0.000 \mathrm{e}+00$
\end{tabular}

Figure 7 - Pressure (left) and velocity (right) contours at different points in time:

$\mathrm{t}=0 \mathrm{c}(\mathrm{a}) ; \mathrm{t}=1.5 \mathrm{e}-5 \mathrm{c}(\mathrm{b}) ; \mathrm{t}=2.4 \mathrm{e}-5 \mathrm{c}(\mathrm{c}) ; \mathrm{t}=4.2 \mathrm{t}-5 \mathrm{c}(\mathrm{d})$

for $p=1553 \kappa \Pi а, T=473 K$.

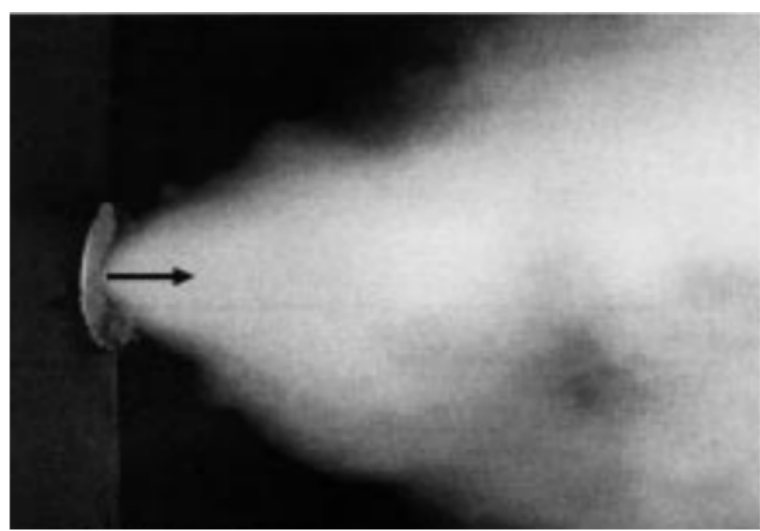

Figure 8 - Pressure outlet from the boiling liquid chamber at a temperature of $473 \mathrm{~K}[10]$

Int. j. math. phys. (Online)

International Journal of Mathematics and Physics 12, №1, 40 (2021) 
The photograph which taken from [9] has been shown in Figure 8, where an experimental jet of boiling liquid can be seen at an initial saturation temperature of $473 \mathrm{~K}$. A comparative analysis of the photograph and the results of numerical experiments shows their qualitative agreement.

\section{Conclusion}

In this paper, we propose a model for the dynamics of a two-dimensional vapor-liquid mixture in the case of a sudden depressurization of a highpressure pipe. The numerical experiments of the model was carried out on the basis of the ANSYS software package. The features of the formation of jets of boiling liquid at various equilibrium initial states of water in a high-pressure vessel under conditions close to the experiments carried out by the authors [10] have been studied. The spatial distributions of pressure, velocity and temperature fields are obtained. The results of numerical experiments have shown that at initial temperatures of the state of water saturation below $473 \mathrm{~K}$, the jet has a conical shape; a further increase in the initial saturation temperature leads to the formation of the initial stage of the jet breakup mode, which is in qualitative agreement with the experimental results of [10]. The results of this work can be used in processing the corresponding experimental data and creating methods for calculating the processes accompanying emergency depressurization of complex multi-element high-pressure systems.

\section{References}

1 Ivandaev A.I., Gubaidulin A.A. Issledovanie nestatsionarnogo istecheniya vskipayushchej zhidkosti $\mathrm{v}$ termodinamicheski ravnovesnom priblizhenii [Investigation of a nonstationary outflow of boiling liquid in a thermodynamic equilibrium approximation]. Teplofizika Vysokikh Temperatur High Temperature vol. 16, no. 3 (1978): 556-562.

2 Edwards A.R., O'Brien T.P. Studies of phenomena connected with the depressurization of water reactors. J. Br. Nucl. Energ. Soc. vol. 9, no. 2 (1970): 125-135.

3 Nigmatulin R.I. Dynamics of multiphase media. Hemisphere, N.Y. vol. 1 (1990): 532.

4 Shagapov V.Sh., Yalaev A.Ya. On the theory of the bulk boiling of a liquid with transition into a metastable state. Theoretical Foundations of Chemical Engineering vol. 46, no. 4 (2012): 348-358.

5 R.Kh. Bolotnova and V.A. Buzina. Spatial modeling of the nonstationary processes of boiling liquid outflows from high pressure vessels. computational continuum mechanics vol. 7 , no 4 (2014): 343-352.

6 Pinhasi G.A., Ullmann A., Dayan A. 1D plane numerical model for boiling liquid expanding vapor explosion (BLEVE). Int. J. Heat Mass Tran. vol. 50, no. 23-24 (2007): 4780-4795.

7 Ivashnev O.E. Specific features of the modeling of boiling-fluid flows. Fluid Dynamics vol. 43, no. 3 (2008): 390-401.

8 Bolotnova R.Kh., Buzina V.A., Galimzianov M.N., Shagapov V.Sh. Gidrodinamicheskie osobennosti protsessov istecheniya vskipayushchej zhidkosti [Hydrodynamic features of the boiling liquid outflow processes]. Teplofizika i aeromehanika - Thermophysics and Aeromechanics vol. 19, no. 6 (2012): 719-730.

9 Pribaturin N.A., Bezrukov Yu.A., Bykov M.A., Krasnov S.N., Onshin V.M., Lezhnin S.I., Sorokin A.L. Issledovanie strui pri istechenii vskipayushchej vody pri razryve truboprovoda [The study of the jet at the outflow of boiling liquid at break pipe]. Proceedings of the 4th Russian National Conference on Heat Transfer vol. 5, no. 6 (2006): 284-287.

10 Reshetnikov A.V., Mazheiko N.A., Begletsov V.N., Skokov V.N., Koverda V.P. Pulsation dynamics during explosive boil-up of overheated water jets. Technical Physics Letters vol. 33, no. 9 (2007): 732-734.

11 Nigmatulin R.I., Bolotnova R.Kh. Widerange equation of state for water and steam: Simplified form. High Temperature vol. 49, no. 2 (2011): 303-306. 